\title{
Materials Handling Equipment Selection Using Integrated Fuzzy AHP and VIKOR Methods
}

\author{
Zoran Bogićević ${ }^{1 *}$, Aleksandar Pejčev ${ }^{2}$, Goran Marković ${ }^{1}$ \\ ${ }^{1}$ Faculty of Mechanical and Civil Engineering in Kraljevo, University of Kragujevac, Kraljevo (Serbia) \\ ${ }^{2}$ University of Belgrade, Faculty of Mechanical Engineering
}

\begin{abstract}
By combining the methods for determining the relative weights of the criteria and standard methods of ranking of alternatives, one makes optimal decisions about a certain issue, regardless of the nature of the parameters that describe it. Selection of materials handling equipment for typical conditions and working environment is one of the problems of multicriteria analysis, i.e. the selection procedure is not sufficiently structured, dependent on broad areas of knowledge, and requires the application of efficient and effective tool for decision making. The proposed methodology of equipment selection is a combination of positive experiences in the application of known methods of decision-making and their modifications (Fuzzy AHP and VIKOR). In this case, process of the forming of system alternatives and defining criteria are illustrated in a numerical example of the equipment (device) selection within the transport and handling mechanization (trucks - forklift).
\end{abstract}

Keywords: material handling equipment, multi-criteria decision making, fuzzy AHP, VIKOR

\section{INTRODUCTION}

Selection of transport-warehouse system equipment, within the system as a logistics center or centralized industrial warehouse, is a problem that requires manipulation with disparate data while including a significant number of relevant criteria and objectives that can most often be in conflict. In this case, transportation is seen as one of the most important activities in the logistic system of physical distribution of goods. Overall, in solving transport problems, especially choice of transport equipment as elements of the supply chain, there is no ideal solution, i.e. certain questions have to be answered before it starts to conceptually solve transport system [9]. So, when setting the terms of reference and the main project of the industrial storage system it is necessary to embrace and implement a series of activities strictly according to the order, which is integrated in several projects and/or subprojects. In that process, the most important tasks are:

- determining of the system location,

- forecasting and defining the type and amount of stock,

- set the storage technology and implementation of design of warehouses,

- designing the structure of the storage system and

- the design and selection of transport and reloading equipment and its installation.

Notable is the conclusion that the application of planning methods of modern storage system is practically implemented through three main phases: the formation of an alternative, the evaluation and selection of alternatives and dynamic analysis of selected alternative.

The procedure of materials handling equipment selection is an important area of decision making in distribution, manufacturing and warehousing (areas in which material handling plays a major role), and at the same time characterized by direct effects on the production and distribution as well as quality services. These direct effects indicate that the process of selecting equipment is generally the basis for strategic decision-making. Selection of materials handling equipment for typical conditions and working environment is one of the problems of multicriteria analysis, i.e. the election procedure is not sufficiently structured, it is dependent on broad areas of knowledge, and requires the application of efficient and effective tool for decision making. By using the project request, the primary technical parameter is used as a starting material for the definition and elaboration of possible variants - alternative solutions. For further exploration and evaluation shall be taken only alternative in which all evaluation criteria satisfactory fulfillment of objectives.

Many heuristic techniques can be used directly in solving the problem of choice of equipment or adapted to this purpose. Meanwhile, the ability and experience of decision-makers in the election procedure can significantly affect the final solution. There is a large number of papers in the literature that focuses on the problems of selection of appropriate material handling equipment, particularly in the areas of manufacturing and warehousing. Just as mentioned above multidimensionality also a large number of different criteria indicates the existence of a large number of different approaches and models for formulating and solving it. Noticeable is the fact that in a number of papers as the most common approach for this purpose is the application of analytical hierarchy process - AHP [4]. The classic technique makes the process of comparison is too complicated and bulky in order to collect the right way reviews the decision maker. In order to eliminate this shortcoming in the comparison at all hierarchical levels is used fuzzy logic, i.e. Fuzzy AHP. Dagdeviren [4] for the purpose of selecting the most suitable equipment uses an integrated approach to AHP and PROMETHEE. These are works in the field of so-called combined or hybrid method (based on the combined application of different methods of making electro, TOPSIS, ANP, etc.) that address the 
equipment selection of which meets the decision maker [3, 12]. In recent years, the problems associated with collective decision-making, subjectivity of decision-makers and the use of qualitative expression and the alternative by individual criteria provide numerous methods based on generalized fuzzy numbers, in the case of the equipment selection $[10,14,15]$ or of the equipment characteristics $[1,11,16]$. Further review of the literature shows that one part of the research in this area aimed at the development of expert systems to support decision making in the selection of appropriate equipment $[2,5,10,13]$. Such systems in order to select the most suitable variant from a set of predefined alternatives, by their function requires the user to enter the desired value of the equipment. In this case, the knowledge base is mostly comprised of rules generated from the literature, documentation, equipment or consultation with experts.

This paper presents a method for forming effective methods and techniques for decision support so that the choice of generalized criteria is not left to experience and subjective view of decision-makers in choosing equipment. Also, in this case it is necessary to point out the need to adequately evaluated and included in the model to determine the best alternative to the specified criteria, all the characteristics and parameters of criteria and whether in quantitative or qualitative form. Convenience, efficiency and applicability of the proposed approach in the selection of electric forklifts with three point necessary for the execution of warehouse tasks, are shown by the analysis of numerical examples.

\section{FUZZY AHP AND VIKOR METHOD}

The final order of the alternatives in the problem of the equipment selection depends on the applied techniques for decision making, the process of defining the criteria for the evaluation and assessment of their relative importance. In the process of determining the relative weight of criteria of subjective decisions are crucial, and the literature is present aspiration to subjective attitude of the weights of criteria (significance) is easier to express the importance of comparing the criteria by pairs than once. Fuzzy logic proved to be excellent in models in which intuition and evaluation of the primary elements. Applying the theory of fuzzy sets was due to frequent handling of decision-makers in terms of vagueness or so called partial truth $[8,9]$. So in the present combined approach of fuzzy AHP was used to determine the relative weights of the criteria, and VIKOR method [7] focuses on the ranking and selection of alternatives at present conflicting criteria, taking advantage of the ideal dot as a reference point in space criterion function.

\subsection{Fuzzy AHP}

Fuzzification of conventional AHP method was carried out by using triangular fuzzy numbers and interval arithmetic to determine the weights of criteria and alternatives, starting from the scale of the relative importance and evaluation in pairs including all necessary matrix operations. Triangular fuzzy numbers are used to improve the process of scaling in the formation of comparison matrix, while fuzzy arithmetic is used to determine the fuzzy vector eigenvalues.The procedure of this approach can be presented in several steps [4,9]:

Step 1: The determination of criteria weights i.e. the relative weights of the two elements at the same level of hierarchy by using triangular fuzzy numbers $(\tilde{1}, \tilde{3}, \tilde{5}, \tilde{7}, \tilde{9})$.

Step 2: The formation of fuzzy comparison matrix $\tilde{A}\left(a_{i j}\right)$ as:

$$
\tilde{A}=\left[\begin{array}{cccc}
1 & \tilde{a}_{12} & \cdots & \tilde{a}_{1 n} \\
\tilde{a}_{21} & 1 & \cdots & \tilde{a}_{2 n} \\
\vdots & \vdots & \cdots & \vdots \\
\tilde{a}_{n 1} & \tilde{a}_{n 2} & & 1
\end{array}\right]
$$

where:

$$
\tilde{a}_{i j}=\left\{\begin{array}{lr}
\tilde{1}, \tilde{3}, \tilde{5}, \tilde{7}, \tilde{9} & i>j \\
1 & i=j \\
\tilde{1}^{-1}, \tilde{3}^{-1}, \tilde{5}^{-1}, \tilde{7}^{-1}, \tilde{9}^{-1} & i<j
\end{array}\right.
$$

Step 3: The determination of fuzzy eigenvalues, which represents the solution of the system:

$$
\tilde{A} \tilde{x}=\tilde{\lambda} \tilde{x}
$$

$\tilde{A}$ is - where A is n x n fuzzy matrix which contains fuzzy numbers $\tilde{a}_{i j}, \tilde{x}_{\text {is a } \mathrm{n}} 1$ fuzzy eigenvector containing the fuzzy numbers $\tilde{x}_{i}$.

Interval arithmetic is used for all operations, i.e. interval arithmetic and methods of $\alpha$-cuts are used for multiplication and addition of fuzzy number, and the equations are:

$$
\begin{aligned}
& \forall \alpha \in[0,1] \\
& \tilde{A} \alpha=[l \alpha, u \alpha]=[(m-l) \alpha+l, u-(u-m) \alpha] \\
& \\
& a_{i 1 l}^{\alpha} x_{i l}^{\alpha}+\cdots+a_{i n l}^{\alpha} x_{n l}^{\alpha}=\lambda x_{i l}^{\alpha} \\
& a_{i 1 u}^{\alpha} x_{i u}^{\alpha}+\cdots+a_{i n u}^{\alpha} x_{n u}^{\alpha}=\lambda x_{i u}^{\alpha} \\
& \tilde{a}_{i j}^{\alpha}= {\left[a_{i 1 l}^{\alpha}, a_{i 1 u}^{\alpha}\right], \tilde{x}_{i}^{\alpha}=\left[x_{i l}^{\alpha}, x_{i u}^{\alpha}\right], \tilde{\lambda}^{\alpha}=\left[\lambda_{l}^{\alpha}, \lambda_{u}^{\alpha}\right] } \\
& \text { for } 0<\alpha \leq 1 \text { и } i=1,2, \ldots, n, j=1,2, \ldots, n .
\end{aligned}
$$

where: $l$-lower limit of fuzzy number, $m$-mean value of fuzzy number and $u$-upper limit of fuzzy number.

The degree of satisfaction can be obtained from decision-maker by index of optimism $\lambda$. The larger the index $\lambda$, the higher the degree of satisfaction [4]:

$$
\tilde{a}_{i j}^{\alpha}=\lambda a_{i j u}^{\alpha}+(1-\lambda) a_{i j l}^{\alpha}, \forall \lambda \in[0,1]
$$

The degree of satisfaction and reconstructed matrix can be estimated by fixing parameter $\alpha$ and setting the index of optimism $\lambda$, as follows:

$$
\tilde{A}=\left[\begin{array}{cccc}
1 & \tilde{a}_{12}^{\alpha} & \cdots & \tilde{a}_{1 n}^{\alpha} \\
\tilde{a}_{21}^{\alpha} & 1 & \cdots & \tilde{a}_{2 n}^{\alpha} \\
\vdots & \vdots & \cdots & \vdots \\
\tilde{a}_{n 1}^{\alpha} & \tilde{a}_{n 2}^{\alpha} & & 1
\end{array}\right]
$$


The five triangular fuzzy numbers are defined with corresponding intensity of importance (Fig. 1).

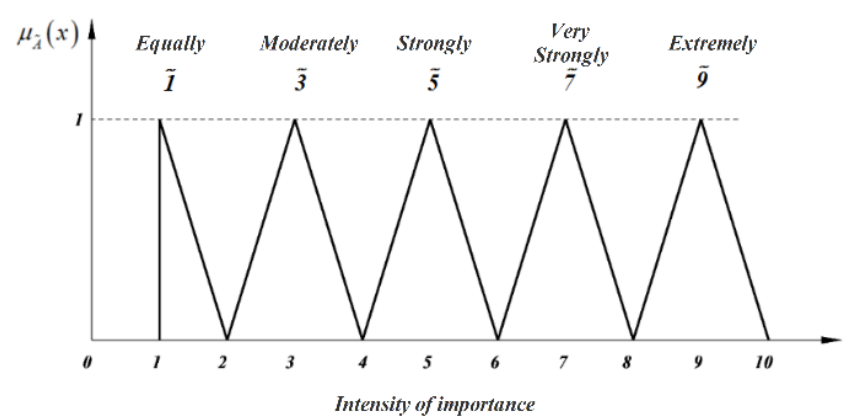

Figure1. Fuzzy membership function

The consistency index (CI) and consistency ratio (CR) are given as follows:

$$
\begin{gathered}
C I=\left(\lambda_{\max }-n\right) /(n-1) \\
C R=C I / R I
\end{gathered}
$$

For the purposes of further research and easier application of the proposed algorithm for obtaining the relative weights of criteria (Fig. 2), the program tool is developed using MATLAB programming. The developed program is integrated at a later stage with a tool developed for the purpose of ranking of alternatives and it is characterized by the ability to use an unlimited number of criteria as well as speed and flexibility.

Besides, the developed tool enables decision-makers to use different values of the confidence level and index of optimism as input in the interval $[0,1]$ and to show their influence on the final results.

Thus, the process of evaluating and determining the criteria preference is implemented as follows:

1. Preparing the input data (number of criteria and alternatives, the value of the confidence level and index of optimism).

2. Generating the fuzzy comparison matrix. Fuzzy comparison matrix is square in size, equal to the number of criteria, values 1 are on the main diagonal, and other values in the form of triangular fuzzy numbers are entered as a result of pairwise comparisons of each criterion on each level based on the scale of five points (Fig. 1). In this step, only direct values are entered while the inverted ones are automatically generated.

3. Applying (4) and (5), the program automatically generates $\alpha$-cut matrix.

4. Normalizing the matrix from the previous step, calculating the fuzzy eigenvalues i.e. finding the relative weights of criteria and consistency index and ratio in accordance with the fuzzy AHP approach. If the consistency ratio is less than 0.10 , the result is sufficiently accurate and there is no need for correction in pairwise judgment and repetition of calculation.

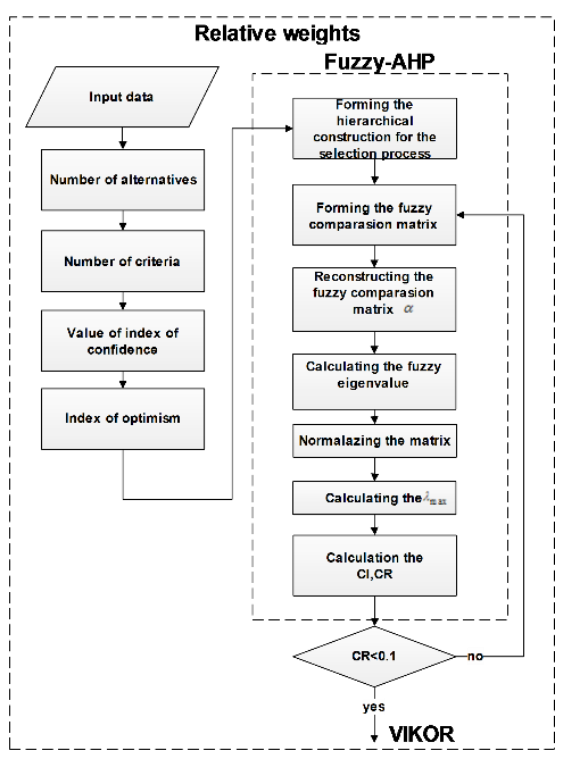

Figure 2. The algorithm for determining the relative weight of criteria

\subsection{VIKOR method}

VIKOR multicriteria method solves the following problem: Determine the best (compromise) solution in multicriteria sense from a set of $J$ permissible alternative $A_{l}$, $A_{2}, \ldots A_{J}$, evaluated according to a set of $n$ criteria functions. Inputs are the elements of the matrix performance where $f_{i j}$ the value of the $i$-th criterion function for an alternative $A j$. This method focuses on the ranking and selection of alternatives at present conflicting criteria, and without using the ideal point as a reference point in space criterion function. However, there is no alternative that meets all the criteria at the same time, so a permissible solution that is closest to the ideal in the space of criterion function is needed. The solution that is closest to the ideal is called a compromise solution based on adopted measures distances [7]. As a measure of distance from the ideal point are used "borderline" metrics $L_{P}$ of compromise programming methods, i.e. the measures $S_{j}$ and $R_{j}$ :

$$
\begin{aligned}
& S_{j}=\frac{\sum W_{i}\left(f_{i}^{*}-f_{i j}\right)}{\left(f_{i}^{*}-f_{i}^{-}\right)} \\
& R_{j}=\max \frac{W_{i}\left(f_{i}^{*}-f_{i j}\right)}{\left(f_{i}^{*}-f_{i}^{-}\right)}
\end{aligned}
$$

where:

$f_{i j}$ - values and these criteria functions for $j$-th variant solution $i=1, \ldots, n \mathrm{i} j=1, \ldots, m$

$f_{i}^{*}=\max f_{i j}$ i $f_{i}^{-}=\min f_{i j}$ if the latter criterion function shows a profit, and

$f_{i}^{*}=\min f_{i j}$ i $f_{i}^{-}=\max f_{i j}$ if $i$-criteria function shows loss; $W_{i} \geq 0$ the weights of selected criteria.

Ranging through measures $S j$ and $R j$ determine the positioning $s\left(V_{j}\right)$ i $r\left(V_{j}\right)$ the ranking of variants $V_{j}, j$ $=1, \ldots, m$. Thus obtained the rank lists differ widely, so a further procedure for the determination of a unified ranking list is needed. This ensured ranking are obtained on the basis of measures $Q j$ :

$$
Q_{j}=v Q S_{j}+(1-v) Q R_{j}, j=1, \ldots, m
$$


where:

$$
Q S_{j}=\frac{S_{j}-S^{*}}{S^{-}-S^{*}} \mathrm{i} Q R_{j}=\frac{R_{j}-R^{*}}{R^{-}-R^{*}}
$$

$S^{*}=\min _{j}, S^{-}=\max S_{j}$,

$R^{*}=\min _{j}, R^{-}=\max R_{j}$

$v$ - weight strategy making " most criteria ", and the value range in the interval $(0,0.5, \mathrm{i} 1)$.

Variant $V_{j}$ is mmultidisciplinary better than $V_{k}$ ranked by $Q$, if it is $Q_{j}<Q_{k}$ and has a higher position in the rankings. Ranking list $Q$ obtained by joining the lists $Q_{R}$ i $Q_{S}$. The ranking is done by sorting varieties according to the values measures $Q_{S}, Q_{R}$ i $Q$. The best solution is one which has the lowest value was measured and it occupies first place in the ranking.

Measure $Q_{j}$ is a linear function of weight strategies "to satisfy most of the criteria" $v$, and the position on the list Q "linear combination" position on the lists $Q_{R}$ i $Q_{S}$. Stability positions variants in the ranking are analyzed by the changing values of weight coefficients. Method VIKOR proposed as the best variant multiple criteria (for adopted values $\mathrm{W}$ ), which is in the first position on the compromise ranking for $v=0.5$ only if they have:

- "sufficient advantage" of a variant of the following positions (condition U1),

- "sufficiently stable" position with the change in weight (the condition $U 2$ ).

For the evaluation of the "benefits " it is used the difference between the measures $Q j$ for $v=0,5$. Variant $V^{\prime}$ has a sufficient advantage over the following $V^{\prime \prime}$ the ranking list if:

$$
Q\left(\mathrm{~V}^{\prime \prime}\right)-Q\left(\mathrm{~V}^{\prime}\right) \geq \mathrm{DQ},
$$

where in DQ, "threshold advantages ", which is determined in relation to the theoretical value Q, $Q \max -Q \min =1-0$, and the number of varieties of $m$ :

$$
\mathrm{DQ}=\min \left(0.25 ; \frac{1}{m-1}\right)
$$

With 0.25 the threshold is limited for cases with a small number of variants. The condition " sufficient benefits " enables the decision maker all variants that are " close " in multicriteria sense. The first variant in the ranking has "sufficiently stable" position if it meets at least one of the following conditions:

- has the first position in the ranking according to Q $v=0,25$ and $v=0,75$,

- has the first position in the ranking according to QS,

- has the first position in the ranking according to $Q R$.

If the first variant of the compromise ranking list does not meet both conditions $U 1$ i $U 2$ it is considered that it is not sufficiently better than the variant with other positions. In such cases, the method VIKOR formed a set of compromises in entering the first variant and a variant behind her. If the first variant does not fulfill a condition $U 1$ then the set of compromise solutions includes only one with the compromise list. However, if does not fulfill only a condition U1 then set of compromise solutions includes versions with compromise ranking lists $V^{\prime}$ i $V^{\prime \prime}, \ldots, V^{(k)}$ which is $Q\left(V^{(k)}\right)-Q(V)<D Q$. [7]

\section{NUMERICAL EXAMPLE}

Therefore, the problem of strategic decision-making, shown in the introduction, is formulated through the process of selection of electrical forklift necessary for the execution of tasks within the warehouse. Defining the alternatives considered problem was carried out with the help of previously developed approaches FAMOD [9], which selects a number from the database, which initially contains data the world's leading manufacturers of this equipment. Elimination module, contains a field for entering the required parameters in the form of questions 8 , wherein the user gives the flexibility that the number of questions to be greater as the number of data in base. It is necessary to introduce requirements for the most important characteristics when choosing a forklift.

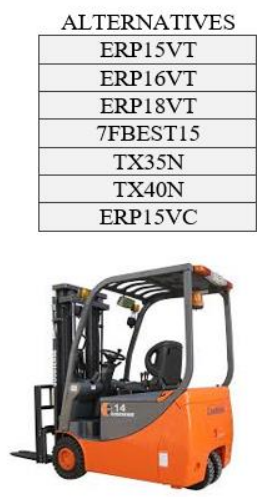

\section{Figure 3. The final number of alternatives which satisfy the performance requirements}

For the following input parameters entered : required load capacity of $1500 \mathrm{~kg}$, lift height $3300 \mathrm{~mm}$, lifting speed with load and no-load $0.450 .55 \mathrm{~m} / \mathrm{s}$ and a turning radius of $1500 \mathrm{~mm}$, overall width $1050 \mathrm{~mm}$, length up to $1700 \mathrm{~mm}$ forks on output screen, eliminating modules is given 7 alternatives which meet the required performance ( Fig. 3). This collection was subsequently transferred to a module designed for determining the relative weights of criteria . For the selection procedure of optimal solution, we used the following criteria: costs: fixed and variable load capacity, speed, lifting height, turning radius, width and safety and ergonomics. The criteria, fixed and variable costs as well as safety and ergonomics are defined by the linguistic expressions, while others are defined as numerical values.

The proposed approach for solving these problems consists of two steps. In the first step, in linguistic expressions are converted fuzzy numbers or fuzzy numbers in the second step, the same converted into real numbers. Since then all the numbers in the matrix of values that alternatives are taken by individual criteria are real numbers. Defuzzification value of fuzzy number is obtained as the mean value of the so-called, maximum and minimum set. By defining the interval scale conversion is carried out converting the linguistic expressions for flexibility in matching triangular fuzzy number, and the corresponding numerical value is automatically assigned to the same as the average of the so-called maximum and minimum set. Since then all the numbers in the matrix of values that alternatives are taken by individual criteria are real numbers. 


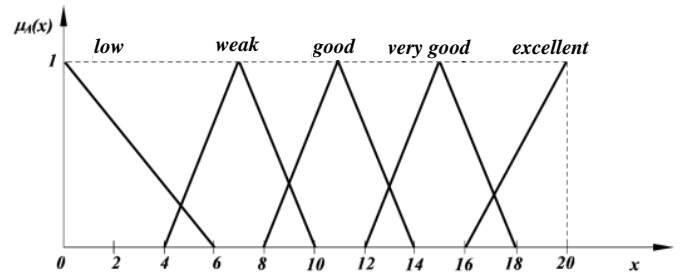

Figure 4. The membership functions of triangular fuzzy number (TFN) safety and ergonomics

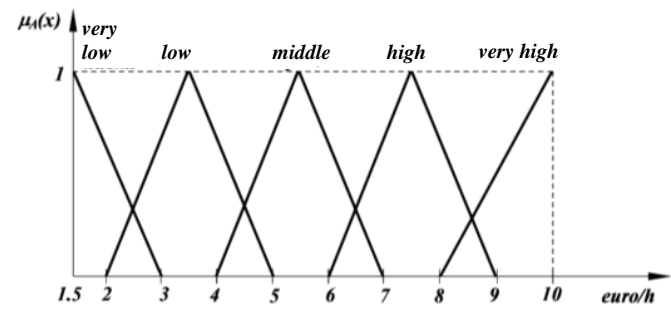

Figure 5. The membership functions for TFN fixed costs

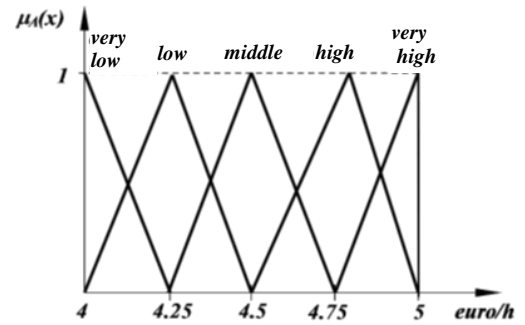

Figure 6. The membership functions for TFN variable costs

Table 1. The comparison of criteria - fuzzy comparison matrix

\begin{tabular}{ccccccccc}
\hline & $\mathbf{K 1}$ & $\mathbf{K} 2$ & $\mathbf{K} 3$ & $\mathbf{K} 4$ & $\mathbf{K 5}$ & $\mathbf{K} 6$ & $\mathbf{K} 7$ & $\mathbf{K} 8$ \\
\hline $\mathbf{K} 1$ & $\tilde{1}$ & $\tilde{5}^{-1}$ & $\tilde{7}^{-1}$ & $\tilde{\tilde{3}}^{-1}$ & $\tilde{5}^{-1}$ & $\tilde{3}$ & $\tilde{3}^{-1}$ & $\tilde{3}^{-1}$ \\
$\mathbf{K} 2$ & & $\tilde{1}$ & $\tilde{3}^{-1}$ & $\tilde{1}$ & $\tilde{3}^{-1}$ & $\tilde{1}$ & $\tilde{3}^{-1}$ & $\tilde{3}^{-1}$ \\
$\mathbf{K} 3$ & & & $\tilde{1}$ & $\tilde{5}$ & $\tilde{5}$ & $\tilde{7}$ & $\tilde{1}$ & $\tilde{3}$ \\
$\mathbf{K} 4$ & & & & $\tilde{1}$ & $\tilde{3}^{-1}$ & $\tilde{1}$ & $\tilde{5}^{-1}$ & $\tilde{3}^{-1}$ \\
$\mathbf{K 5}$ & & & & & $\tilde{1}$ & $\tilde{5}$ & $\tilde{3}^{-1}$ & $\tilde{1}$ \\
$\mathbf{K} 6$ & & & & & & $\tilde{1}$ & $\tilde{1}$ & $\tilde{5}^{-1}$ \\
$\mathbf{K} 7$ & & & & & & & $\tilde{1}$ & $\tilde{3}$ \\
$\mathbf{K} 8$ & & & & & & & & $\tilde{1}$ \\
\hline
\end{tabular}

Table 2. Vector eigenvalues of matrix comparison criteria

\begin{tabular}{ccccccccc}
\hline Criteria & K1 & K2 & K3 & K4 & K5 & K6 & K7 & K8 \\
\hline K1 & 0.047 & 0.064 & 0.044 & 0.118 & 0.039 & 0.056 & 0.044 & 0.045 \\
K2 & 0.041 & 0.051 & 0.044 & 0.118 & 0.039 & 0.056 & 0.077 & 0.045 \\
K3 & 0.332 & 0.359 & 0.305 & 0.197 & 0.334 & 0.222 & 0.272 & 0.379 \\
K4 & 0.017 & 0.018 & 0.062 & 0.039 & 0.023 & 0.133 & 0.044 & 0.045 \\
K5 & 0.142 & 0.154 & 0.108 & 0.197 & 0.111 & 0.222 & 0.077 & 0.158 \\
K6 & 0.041 & 0.045 & 0.062 & 0.014 & 0.023 & 0.044 & 0.077 & 0.045 \\
K7 & 0.237 & 0.154 & 0.267 & 0.197 & 0.334 & 0.133 & 0.218 & 0.158 \\
K8 & 0.142 & 0.154 & 0.108 & 0.118 & 0.097 & 0.133 & 0.190 & 0.126 \\
\hline
\end{tabular}

Table 3. Relative weights of criteria

\begin{tabular}{cc}
\hline Relative weights & Criteria \\
\hline 0.057 & $\mathrm{~K} 1$ \\
0.059 & $\mathrm{~K} 2$ \\
0.300 & $\mathrm{~K} 3$ \\
0.048 & $\mathrm{~K} 4$ \\
0.146 & $\mathrm{~K} 5$ \\
0.044 & $\mathrm{~K} 6$ \\
0.212 & $\mathrm{~K} 7$ \\
0.134 & $\mathrm{~K} 8$ \\
\hline
\end{tabular}

Table 4. The maximum eigenvalue, the consistency index and ratio

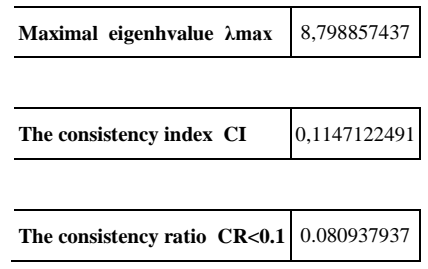

Table 5. Inputs - the values of alternative matrix

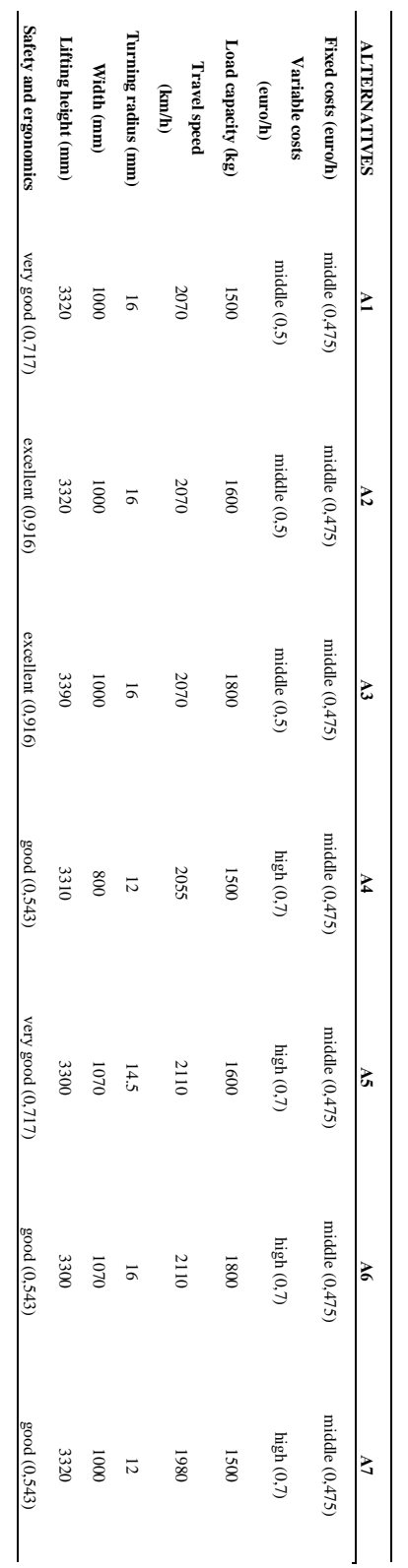

Table 6. Comparative analysis of the different methods of multi-criteria analysis results

\begin{tabular}{cccc}
\hline Alternative & VIKOR & TOPSIS & FAMOD \\
\hline A1 & 5 & 4 & 3 \\
\hline A2 & 3 & 2 & 2 \\
\hline A3 & 1 & 1 & 1 \\
\hline A4 & 7 & 7 & 6 \\
\hline A5 & 4 & 3 & 7 \\
\hline A6 & 2 & 5 & 4 \\
\hline A7 & 6 & 6 & 5 \\
\hline
\end{tabular}

For determining the final ranking of alternatives presented for input parameters, by the developed tool RTK, one found respectively the relative weight of the criteria and 
the level of consistency in accordance with the present steps fuzzy AHP approach (Table 1, 2 and 3). If the degree of consistency is less than 0.10 , the result is sufficiently accurate and there is no need for adjustments in comparisons and repetition budget (Table 4). In the next phase, the previously defined alternatives are evaluated on the basis of the adopted criteria and their weighting factors.

Using MATLAB, to obtain results with the help of VIKOR it is afforded a ranking of alternatives in the process of election equipment. In order to confirm the results obtained by the combined approach as well as proof of applicability and practicality of the same, the discussed problem is analyzed using standard and modified methods of multi-criteria analysis (TOPSIS and FAMOD method). The results are shown in Table 6 . The decision maker in the observed case, makes the final decision on the selection of equipment that is conceptual solution (alternative 3) exceeds all present potential limitations and represents the best solution. In all cases the most optimal variant remains unchanged, indicating the robustness of the proposed approach in resolving these types of multicriteria tasks.

\section{CONCLUSION}

Defined approach is a combination and expansion of some so far formulated models, both for determining the relative weights and the ranking of alternatives considered problem, without sensitivity analysis of the final order of alternatives due to changes in weight coefficients criteria. In literature and in pursuit of this approach is that the methods offices and possibly standardize, where the main assumption of which goes to the subjective attitude on the relative weights of the criteria, it is easier to express the importance of comparing the criteria by pairs rather than all at once. Setting a value criterion weight is a particular problem and its solution depends on the structure of preferences of decision-makers and the manner of its expression and formulation. Besides the shown method gives the possibility that the decision-maker uses as input different values of the index conviction and optimism in the interval $[0,1]$ and show their influence on the final results. The possibility of taking into account the linguistic expression of alternative values and reduce the number of criteria for operational and acceptable level are the directions in which they should go further research in order to improve the given approach and the establishment of comprehensive tools in solving a wide range of realistic and technical problems.

\section{REFERENCES}

[1] Ayang, Z., Ozdemir, R. G.: A fuzzy AHP approach to evaluating machine tools alternatives, Journal of Intelligent Manufacturing, 17,179-190, 2006

[2] Chan, F.T., Ip, R. W. L., Lau, H.: Integration of expert system with analytic hierarchy process for the design of material handling equipment selection system, Journal of Materials processing Technology, 116, 137-145, 2001
[3] Chang, C.-W.: Collaborative decision making algorithm for selection of optimal wire saw in photovoltaic wafer manufacture, Journal of Intelligent Manufacturing, 23 (2012), 533-539, 2012, doi:10.1007/s10845-010-0391-6

[4] Dagdeviren, M.: Decision Making in equipment selection: an integrated approach with AHP and PROMETHEE, Journal of Intelligent Manufacturing, 19,397-406, 2008.

[5] Fonseca, D. J., Uppal,G., Greene, T. J.: A knowledge-based system for conveyor equipment selection, Expert Systems with Applications, 26, 615-623, 2004

[6] Kulak, O.: A decision support system for fuzzy multi-atribute selection of material handling equipments, Expert Systems with Applications 29(2005), 310-319, 2005

[7] Lucien Duckstein and Serafim Opricovic (1980) "Multiobjective Optimization in River Basin Development”, Water Resources Research, 16(1), 14-20

[8] Marković, G., Gašić, M., Kolarević, M., Savković, M., Marinković., Z.: Application of the MODIPROM method to the final solution of logistics centre location, Transport 28(4), pp. 341351, 2013.

[9] Marković, G.: Model regionalne logistike transportnim sistemima, Doktorska disertacija, str.183, Kraljevo, 2014.

[10] Kulak, O.: A decision support system for fuzzy multi-atribute selection of material handling equipments, Expert Systems with Applications 29(2005), 310-319, 2005

[11] Rai, R., Kameshwaran, S., Tiwari, M.K.: Machine tool selection and operation allocation in FMS: solving a fuzzy goal programming model using a genetic algorithm, International Journal of Production Research,40, 641-645, 2002

[12] Stam, A., Kuula, M.: Selecting a flexible manufacturing system using multiple criteria analysis, International Journal of Production Research, 29, 803-820, 1991

[13] Tabucanon, M. T., Batanov, D. N.,Verma, D.K.: Intelligent decision support system (DSS) for the selection process of alternative machines for flexible manufacturing systems (FMS), Comuters in Industry, 25, 131-143, 1994

[14] Tuzkaya, G., Gulsun, B., Kahraman, C. Ozgen, D.: An integrated fuzzy multi-criteria decision making methodology for material handling equipment selection problem and an application, Expert Systems witjh Applications 37 (2010), 2853-2863, 2010

[15] Wang, T. Y., Shaaw, Ch. F., Chen, Y. L.: Machine selection in flexible manufacturing cell: A fuzzy multiple attribute decision making approach, International Journal of Production Research,38, 2079-2097, 2000

[16] Yurdakul, M., Tansel, I. Y.: Analysis of the benefit generated by using fuzzy numbers in a TOPSIS model developed for machine tool selection problems, Journal of Material Processing Technology, 209, 310-317,2009. 